Мясоедов А.И.

Природа финансовых циклов и их роль в развитии кризисных процессов на примере Украины

Московский Государственный Психолого-Педагогический Университет, Россия, Москва, ул. Сретенка, д. 29

e-mail: retvil@mail.ru

Научный руководитель: Иванова С. П., кэ.н., доцент кафедры «Теория и практика управления» института «Иностранные языки, современные коммуникации и управление» Московского государственного психологопедагогического университета

\begin{abstract}
Аннотация
В статье описана сущность финансовых циклов и особенности их проявления: более длительный период по сравнению с экономическими циклами, следующий за фазой кризиса после пика цикла, более тяжелая рецессия. Определены ключевые показатели финансового цикла: кредитные составляющие (отношение кредитов к ВВП, темпы роста кредитных ресурсов, отношение кредитов к активам банковской системы) и динамика цен на недвижимость. Рассмотрена динамика этих проявлений на примере Украины по показателям и направлениям минимизации влияния финансового цикла.

Ключевые слова: финансовый цикл; деловой цикл; показатели финансового цикла; банковская система; отношение кредита к ВВП; финансовый кризис.
\end{abstract}

Информация для цитирования: Мясоедов А.И. Природа финансовых циклов и их роль в развитии кризисных процессов на примере Украины // Научный результат. Экономические исследования. 2020. Т.6. № 1. С. 24-34. DOI: 10.18413/2409-1634-2020-6-1-0-3

\title{
A.I. Myasoedov \\ The nature of financial cycles and their role in the development of crisis processes on the example of Ukraine
}

Moscow State Psychological and Pedagogical University, 29 Sretenka street, Moscow, Russia

$$
\text { e-mail: retvil@mail.ru }
$$

Scientific Adviser: Ivanovna S.P., PhD in Economics, Associate Professor of the Department of Management Theory and Practice at the Institute of Foreign Languages, modern Communications and Management, Moscow State University of Psychology and Education 


\begin{abstract}
The article describes the essence of financial cycles and features of their manifestation: a longer period in comparison with economic cycles, following the phase of the crisis after the peak of the cycle, a more severe recession. The key indicators of the financial cycle are identified: credit components (the ratio of loans to GDP, the growth rate of credit resources, the ratio of loans to assets of the banking system) and the dynamics of real estate prices. The dynamics of these manifestations on the example of Ukraine in terms of indicators and directions of minimizing the impact of the financial cycle is considered.
\end{abstract}

Key words: financial cycle; business cycle; financial cycle indicators; banking system; credit-to-GDP ratio; financial crisis

Information for citation: A.I. Myasoedov (2020), "The nature of financial cycles and their role in the development of crisis processes on the example of Ukraine", $R e$ search Result. Economic Research, 6 (1), 24-34, DOI: 10.18413/2409-1634-2020-6$1-0-3$

\section{Введение}

Современная экономическая ситуация характеризуется повышением динамичности условий функционирования, высоким уровнем неопределенности, значительным усложнением бизнес-процессов. Финансовый кризис показал неспособность финансового сектора противостоять негативным тенденциям.

Особенности современного этапа социально-экономического развития определяют формирование новых требований в теоретическом осмыслении проблем циклического развития и финансовых кризисов. Широкий спектр научных работ по данной тематике демонстрирует устойчивый интерес к ней представителей практически всех экономических течений. Такой интерес предполагает тщательное изучение и оценку существующего исследовательского потенциала в контексте устойчивости проблем динамики экономической системы и ее финансовой архитектуры, обострившихся под влиянием недавнего финансовоэкономического кризиса.

Таким образом, мировой финансовый кризис 2007-2008 годов выявил необходимость анализа финансовой устойчивости и причин возникновения финансовых кризисов. В этом контексте на первое место выходит макропруденциальная политика, основной целью которой является поддержа- ние финансовой стабильности. Недавний опыт стресса в еврозоне страны в более широком контексте глобального финансового кризиса иллюстрирует системный риск, присущий фазе наращивания и коррекции кипучих финансовых циклов. Отметим, что возможность наложения циклов разной длительности, масштаба и ареалов существования значительно усложняет их дифференциацию и выявление структурных механизмов взаимодействия [Иванова С.П., 2015].

На протяжении длительного этапа развития экономической мысли финансовые факторы экономической нестабильности лишь изредка оказывались в центре внимания исследователей. Следует отметить, что финансовые циклы менее изучены, чем их аналоги делового цикла, хотя в последнее время исследования в этой области расширяются. Хотя предыдущие исследования финансовых циклов были сосредоточены на их эмпирическом изучении, все еще остается много открытых вопросов, особенно в отношении характеристик стран, имеющих отношение к применению макропруденциальной политики на национальном уровне, а также взаимосвязи финансового цикла и кризисов [Иванова С.П., Литвинов А.Н., 2016.].

Вопросам, затронутым в данной работе, в отечественной литературе не уделяется должного внимания. Что касается зарубеж- 
ных исследований, то изучением финансовых циклов, их показателей, продолжительности занимались такие экономисты, как С. Деткен, Ф. Сметс, С. Гудхарт, Б. Гофман, М. Шулярик, А. Тейлор, С. Борио, П. браке, П. Лоу, М. Дреманн, л. Алесси, т. НГ и другие. В своих исследованиях авторы подчеркивают, что чрезмерный рост кредитования является одним из лучших предикторов кризиса. М. Дреманн и С. Борио пытаются определить финансовый цикл для США и других выбранных стран [Borio, C. 2012; Borio, C., \& M. Drehmann 2009; Drehmann, M., Borio, C. and Tsatsaronis, K., 2012]. Они предлагают оценивать финансовый цикл путем объединения кредитных и имущественных цен. С. Борио изучает стилизованные особенности финансового цикла и говорит, что он имеет большую продолжительность и большую амплитуду, чем традиционный деловой цикл [Мясоедов А.И., 2018]. Кроме того, К. Борио утверждает, что большинство банковских кризисов, как правило, сопровождаются быстрой кредитной экспансией, продолжающейся вблизи пика финансового цикла. Аналогичного мнения придерживаются В. Шуларик и А. Тейлор, которые считают, что кредитные агрегаты дают информацию о вероятности будущих финансовых кризисов и что последние следует рассматривать как "неудавшиеся кредитные бумы” [Schularick, M. and Taylor, A., 2012].

В целом особое внимание уделяется движителям финансового цикла и их детерминантам в развитых странах [Borio, C., 2012; Comin, D. and Gertler, M., 2006; Hiebert, P., Klaus, B., Peltonen, T., Schüler, Y. and Welz, P., 2014]. Между тем, за рамками исследования часто остаются факторы, влияющие на глубину финансовых циклов в развивающихся экономиках и их способность адаптироваться к новым условиям функционирования и трансформации ресурсов в условиях циклического экономического развития. Ибо дальнейшее расширение знаний о финансовых циклах, происходящих по мере глобализации и интеграции, невозможно без наращивания потенциала адаптивности национальной экономики к цикличности.

Цель и задачи исследования - обобщить информацию о финансовом цикле и выяснить его взаимосвязь с экономическим кризисом.

\section{Основная часть}

Финансовый кризис вызвал новый интерес к макропруденциальной политике как основе для решения проблемы стабильности финансовой системы в целом, а не только ее отдельных компонентов. Кризис показал нам, что, хотя он и является одной из глобальных целей, поддержание финансовой стабильности имеет более важное значение в контексте сильных и глубоких финансовых отношений [Иванова С.П., Садыкова K.В., 2017].

Хотя существует много литературы, анализирующей различные аспекты развития финансового рынка, общее понимание финансовых циклов все еще ограничено. Это свидетельствует о том простом факте, что большая часть литературы изучает только отдельные аспекты финансовых циклов. Например, одним из исследований является изучение последствий только бумов в ценах активов и кредитов, а не рассмотрение полных циклов на этих рынках. Другие сосредоточены на финансовых кризисах во многих отношениях только в фазах спада циклов [Иванова С.П., Садыкова К.В., 2018].

В отличие от циклических движений в реальном секторе экономики (деловой цикл), для финансового сектора не существует никаких “естественных" показателей цикла. По сравнению с деловыми циклами финансовые циклы развиваются в среднесрочной перспективе, и их анализ выходит за рамки краткосрочной направленности теории деловых циклов. Циклические колебания финансовых переменных могут усиливать экономические колебания, вызывать дисбалансы, приводить к макроэкономической дестабилизации и/или угрожать финансовой стабильности [Романова К.С., 2016]. 
В целом, в литературе нет единого мнения о природе финансового цикла, общепринятого определения финансового цикла не существует.

Самое близкое определение - " самопроизводящая взаимосвязь стоимости активов, рисков, финансовых ограничений привела к буму, а затем и падению рынков" [Borio C., 2012]. В отличие от бизнесциклов, не существует очевидной "естественной” меры финансового цикла [Borio, C., \& M. Drehmann, 2009].

В современной литературе широко описан финансовый цикл, но в то же время трудно найти соответствующий показатель [Aikman, D., Haldane, A. and Nelson, B.: 2015; Behn, M., Detken, C., Peltonen, T. and Schudel, W.: 2013; Breitung, J. and Eickmeier, S.: 2014]. Финансовые циклы можно отличить от деловых циклов по их амплитуде и частоте. Финансовые циклы развиваются в среднесрочной перспективе, и их анализ должен выходить за рамки краткосрочной направленности теории делового цикла. Это означает, что завершение полного цикла от пика до впадины может длиться до десятилетий [Савельева А.B., 2019; Aikman, D., Haldane, A. and Nelson, В.: 2015]. С. Борио определяет финансовые циклы как «самоподдерживающиеся взаимодействия между восприятием ценности и риска, отношением к риску и ограничениями финансирования, которые переходят в бумы, за которыми следуют спады» [Borio, C., 2012]. Эти взаимодействия могут усиливать экономические колебания, вызывать дисбаланс и приводить к макроэкономической дестабилизации и/или угрожать финансовой стабильности [Borio, C., \& M. Drehmann, 2009].

Одна часть литературы описывает финансовые циклы косвенно и получает выводы о том, что финансовые циклы не являются их соответствующими аналитическими целями. Исследования связывают финансовые показатели, такие как цены активов или кредитные агрегаты, с экономической деятельностью [Behn, M., Detken, C., Peltonen, T. and Schudel, W.: 2013; Bernanke, B., Gertler, M. and Watson, M.: 1997; Borio, C., 2012;
Claessens, S., 2014; Claessens, S., Kose, M. and Terrones, M., 2012]. Другие используют финансовые факторы в качестве важных индикаторов в первичном предупреждении [Borio, C., 2012; Drehmann, M., Borio, C. and Tsatsaronis, K.: 2012; Hiebert, P., Klaus, B., Peltonen, T., Schüler, Y. and Welz, P., 2014]

Прямой путь к характеристике финансовых циклов начался после глобального финансового кризиса. Например, Д. Айкман исследует характеристики кредитного цикла в 14 развитых странах за длительный период (1870-2008 гг.) [Aikman, D., Haldane,

A. and Nelson, B., 2015]. Другие исследования, такие как С. Классенс, анализируют циклические изменения цен на кредиты, жилье и акции в 21 развитой стране с 1960 по 2007 год [Claessens, S., 2014]. Заметим, что оба анализа свидетельствуют о высокой синхронности отдельных рядов, в частности между циклом кредитования и циклом цен на жилье. Расследование, проведенное М. Дреманн является первой попыткой построить синтетический показатель финансового цикла, объединив среднесрочные колебания финансовых переменных для семи развитых стран с 1960 по 2011 год [Drehmann, M., Borio, C. and Tsatsaronis, K., 2012]. Группировка кредитных агрегатов и цен на жилье работает хорошо, в то время как цены на акции, как правило, разрушительны, а не выгодны. Они также показывают, что амплитуда и продолжительность финансового цикла увеличились с середины 1980-х гг. Д. Айкман, М. Дреманн демонстрируют тесную связь между пиками финансовых циклов и системными банковскими кризисами [Aikman, D., Haldane, A. and Nelson, B., 2015; Drehmann, M., Borio, C. and Tsatsaronis, K.: 2012].

Несмотря на то, что в литературе используются различные метрики, она дает сходные выводы и выводы: по сравнению с деловыми циклами финансовые циклы, как правило, имеют более высокую амплитуду и более низкую частоту.

Тесно связанные с литературой по финансовым циклам, исследования также связаны с макропруденциальной политикой 
[Холикова Г.М., 2018]. Все большее количество литературы посвящено изучению эффективности циклического движения кредитных мер (например, разрыв между кредитом и ВВП) для определения антициклической буферной ставки капитала [Behn, M., Detken, C., Peltonen, T. and Schudel, W., 2013; Kaminsky, G. and Reinhart, C., 1999]. Обратите внимание, что антициклический буфер используется в случае более чем 1 стандартного отклонения от тренда. Если отклонение больше, следует принять активные меры по снижению этого показателя.

Циклическое движение этого кредитного показателя используется в качестве индикатора раннего предупреждения для выявления нарастания финансовой уязвимости, хотя предсказательная сила различных показателей кредитного агрегата варьируется [Claessens, S., Kose, M. and Terrones, M.: 2012; Panetta, F., 2013].

Наблюдение за ростом кредитования также актуально для целей сохранения финансовой стабильности: в Базеле III предлагается ввести антициклический буфер капитала для защиты банковской системы и экономики от периодов дополнительного роста или замедления кредитования. Было также высказано предположение о том, что разрыв в соотношении кредитов к ВВП является показателем чрезмерного роста кредитования. Разрыв в соотношении кредитов к ВВП определяется как отклонение банковских кредитов-выраженное как отношение к ВВП-от его долгосрочного тренда и, таким образом, само является мерой финансового цикла и показателем финансового рычага [Радостева М.В., 2018].

Проведен критический обзор литературы по финансовому циклу, позволяющий выделить следующие особенности:

- наиболее точно наше положение в финансовом цикле показывают цены на недвижимость и стоимость кредита. Кредитование особенно важно при строительстве и покупке недвижимости, так что эти два компонента обычно взаимосвязаны. Цены акций находятся с двумя ориентирами гораздо меньше корреляции;
- также важны процентные ставки при изучении циклов, волатильности, премии за риск, плохой кредит и т.д.;

- финансовые циклы сменяются реже, чем деловые;

- сразу после пика финансового кризиса должен быть кризис. Обычно, как только цикл достигает своей высшей точки, начинается банковский кризис;

- рецессия после финансового кризиса хуже, чем после экономического. Обычно рецессия на 50\% глубже, чем падение, вызванное экономическим циклом;

- кризис можно предсказать. Современная теория финансовых циклов позволяет обнаружить признаки кризиса в будущем. Более того, риск можно определить достаточно точно и в режиме реального времени. Наиболее четким ориентиром является как положительный показатель отклонения кредита от ВВП, так и цены активов, в частности недвижимости, от исторических норм;

- время цикла зависит от политики государства: чем слабее Фискальная политика, тем сильнее восходящая и нисходящая части цикла.

Анализ моделей, посвященных вопросам прогнозирования финансовых кризисов, позволил выделить три основных аспекта:

1. Финансовые бумы не только предшествовали кризису, но и стали его причиной. Кризис является следствием уязвимости, которая возникает в период бума.

2. Именно кредиты и долги в целом являются двигателем любого бума, ведь компания может позволить себе больше тратить и покупать. Это приводит к неправильному распределению ресурсов, причем, как капитала, так и рабочей силы. Как только цена активов и денежные потоки начинают снижаться в стадии рецессии, долги превращаются в показатель, сдерживающий восстановление.

3. Согласно теории финансовых циклов, инфляция может быть стабильной, но производство при этом будет уменьшаться или увеличиваться, что связано с финансовыми дисбалансами. 
Таким образом, важность изучения и прогнозирования финансового цикла не вызывает сомнений. Более того, в современных условиях функционирования украинской экономики, вызванных действием внешних и внутренних шоков, к которым экономика оказалась не готова, в силу незавершенности важных процессов рыночной трансформации, сильной зависимости от внешних условий, монетизации и долларизации и опережающего роста финансового сектора в отрыве от реального, этот вопрос весьма актуален.

На основе анализа литературы и с учетом показателей финансового цикла сделан анализ его динамики на примере Украины [Мирошин А.В., 2012]. Следует отметить, что его реализация в нашей стране будет носить специфический характер, особенно в последние годы в связи с финансовой, экономической и политической нестабильностью.

Финансовая система Украины накануне финансового кризиса 2008-2009 годов имела все признаки бума. Признавая влияние многих факторов на развитие кризисных процессов в Украине, в том числе системную, циклическую роль как движущей силы негативных тенденций на кредитном рынке, принимая во внимание перенос циклических шоков с внешних финансовых рынков, нельзя переоценить значение этих факторов.

Для того чтобы рассмотреть основные стилизованные особенности украинского финансового цикла, прежде всего необходимо остановиться на соотношении кредитВВП, показано на рисунке 1.

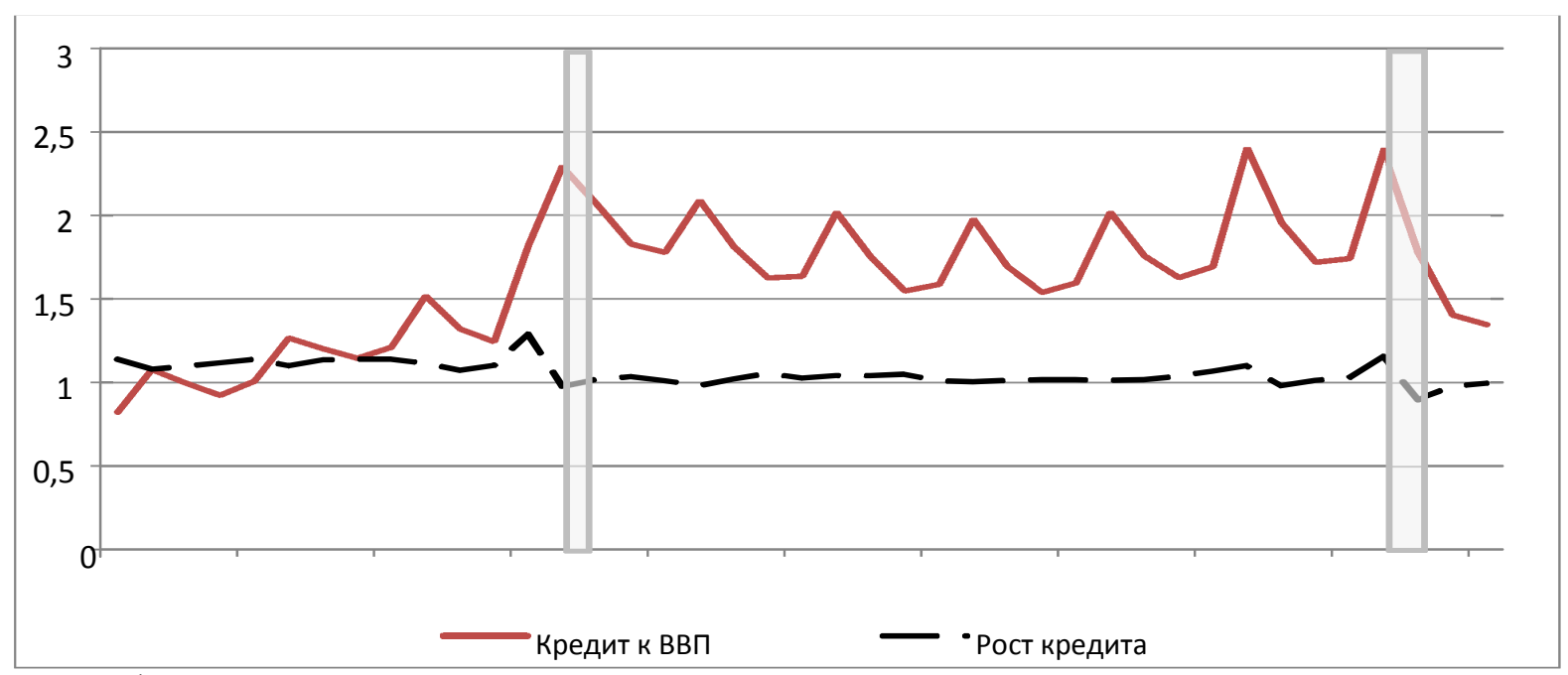

Рис. 1. Циклические изменения финансовых показателей на примере Украины Fig. 1. Cyclical changes in financial indicators on the example of Ukraine

Данные на рис. 1 показывают значительные колебания основных показателей финансового цикла-отношения кредитов к ВВП. Такая динамика свидетельствует о значительной подверженности финансовой системы Украины кризису. Анализ показал, что в конце 2008 года, после пика финансового цикла, в Украине произошел кризис. Такая ситуация была обусловлена влиянием внешних факторов и влиянием многих внешних факторов и разворачивающимся в этот период мировым финансовым кризи- сом. Серые затененные области на рисунке 1 отражают периоды финансового кризиса.

Данные на рис. 1 показывают циклические составляющие отношения кредита к ВВП и роста кредитования. Обе группы помогают нам охарактеризовать основные показатели и сделать заявления об их потенциальной полезности. Очевидным недостатком этого исследования является ограниченное число полных циклов, включенных в этот период времени. 
Графическое исследование показателей финансового цикла не дает убедительного указания на то, какую меру финансового цикла следует выбрать, но оно дает некоторое представление о том, что переменные банковского сектора, по-видимому, имеют важное значение для моделирования финансовых циклов.
В общей сложности мы используем пять потенциальных мер финансового цикла (рис. 2) с различными компонентами. Все показатели цикла включают основной компонент(ы), но также варьируются в зависимости от дополнительных переменных, рассматриваемых в анализе.

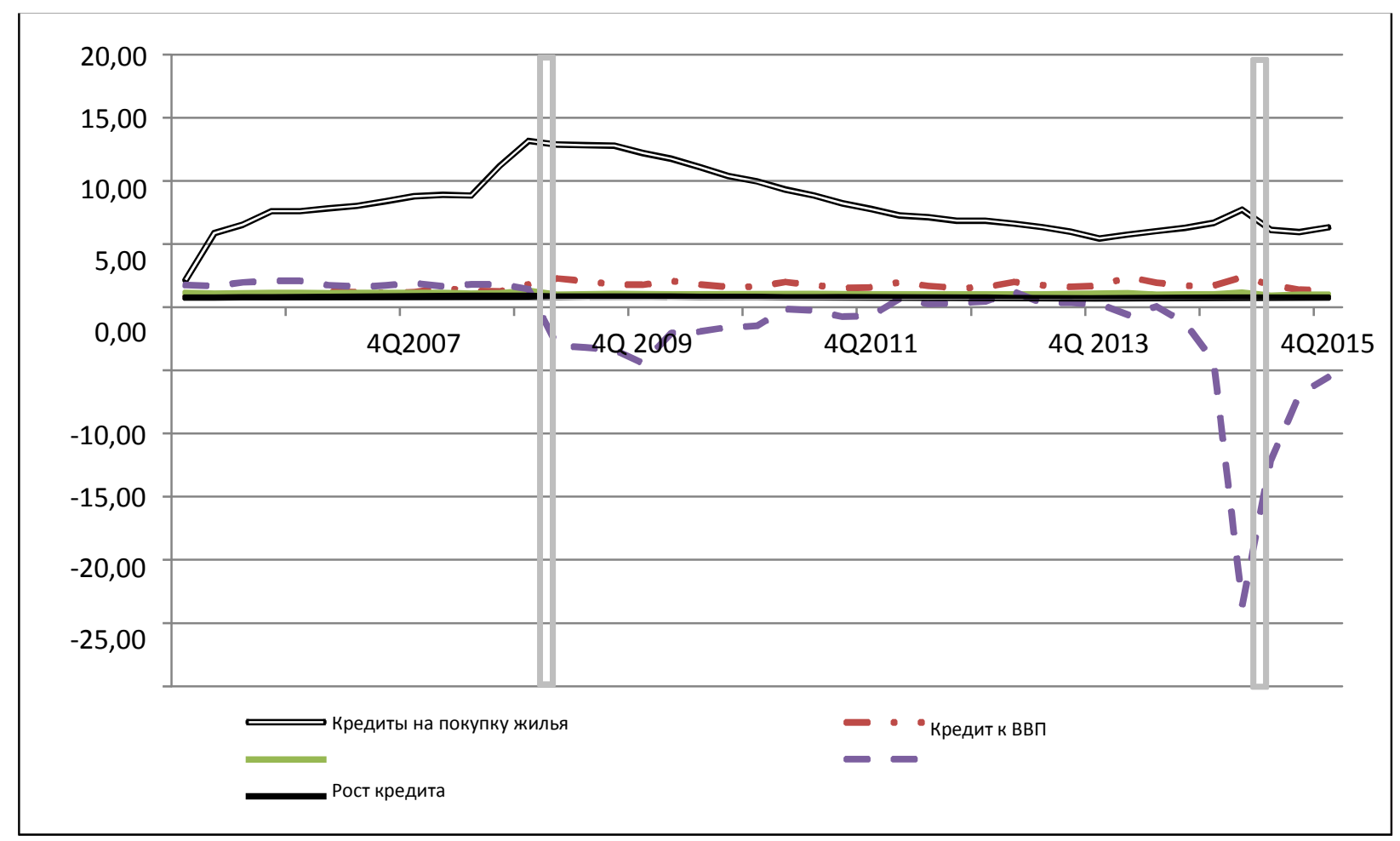

Рис. 2. Динамика показателей финансового цикла на примере Украины Fig. 2. Dynamics of financial cycle indicators on the example of Ukraine

В недавней макропруденциальной литературе высказываются в пользу использования этого показателя, утверждая, что отфильтрованный кредит к временным рядам ВВП полезен для прогнозирования финансовых кризисов и что объяснительная сила может быть увеличена только постепенно путем добавления дополнительных показателей. Следует отметить, что не всякое увеличение кредитования является формой кредитного бума. К интенсификации кредитных процессов может привести, как усиление финансового развития и соответствующее увеличение финансовой глубины, так и "нормальные" циклические тенденции, связанные с подъемом экономики, повышением ее спроса на кредит. Однако именно стремительный рост связан с чрезмерным усилением финансовых дисбалансов и тяготеет к финансовому кризису [Радостева M.B., 2018].

Поворотные точки происходят в разные моменты времени, и амплитуды мер цикла, как правило, различаются. Все показатели финансового цикла (рис.1-2) имеют схожую динамику. Это сходство объясняется тем, что меры имеют некоторые общие компоненты. Пики показателей цикла, повидимому, связаны с периодами финансового кризиса, хотя не каждый пик связан с финансовым кризисом. Рисунок 2 также подтверждает ограниченность данных, поскольку не все показатели финансового цикла доступны в Украине. 


\section{Заключение}

Из проведенного анализа можно сделать следующие выводы:

- финансовая система Украины имеет признаки финансового кризиса. В 2006-2007 годах наблюдалось расширение кредитования, и в этот период были заложены основы для следующего серьезного финансового спада и длительных периодов нестабильного экономического спада [Савельева А.В., 2018];

- из-за недостаточного развития финансового рынка с точки зрения необходимого уровня институциональной поддержки, отсутствия должного регулирования этих процессов со стороны Центрального банка не было достигнуто качественное распределение кредитов и не были внедрены принципы макропруденциального регулирования;

- динамика показателей финансового цикла свидетельствует о неуклонном увеличении емкости отображения не устойчивых финансовых процессов со второго квартала 2006 года;

- на основании динамики определено, что пик отклонения циклических составляющих тренда приходится на конец 2008 г. и середину 2019 г.;

- исходя из закономерностей циклических финансовых процессов можно отметить наличие соответствующих негативных эффектов нерегулируемой кредитной экспансии по длительности и амплитуде спада [6];

- необходимо бороться с кредитным бумом с помощью фискальной, денежнокредитной и макроэкономической политики. Это позволит сдерживать развитие дисбалансов, а также быстро справляться с последствиями. Таким образом, правительство может устранить так называемую "чрезмерную гибкость" системы [Мясоедов А.И., 2017].

В качестве одного из эффективных методов выступает повышение резервных требований и ликвидности банков, например, в рамках Базеля III, но не в период кризиса, а в период бума. Также очень важно, чтобы центральные банки при проведении денежно-кредитной политики руководствовались не только инфляцией, но и другими показателями финансового рынка.

\section{Список литературы}

1. Иванова С.П., 2015. Основные механизмы антикризисного управления по стабилизации банковской системы // Проблемы экономики, организации и управления в России и миpe. Материалы VIII Международной научнопрактической конференции. 2015: 75-76.

2. Иванова С.П., Литвинов А.Н., 2016. Показатели эффективности реальных инвестиций: эволюция и проблемы использования // Вестник Российского экономического университета им.Г.В. Плеханова. 2016. - 5 (89): 73-80.

3. Иванова С.П., Садыкова К.В., 2018. Актуальные вопросы деятельности интегрированных банковских структур // Вестник Российского экономического университета имени Г. В. Плеханова. - 2018. - 1 (97): 90-99.

4. Иванова С.П., Садыкова К.В., 2017. Особенности государственного корпоративного и финансового контроля над деятельностью интегрированных банковских групп // Вестник Российского экономического университета имени Г. В. Плеханова. - 2017. - 5 (89): 62-71.

5. Мирошин А.В., 2012. Факторы, влияющие на негативную мотивацию в период кризиса // В сборнике: Экономические и социальные проблемы глобальной мировой финансовой системы Сборник статей участников III Международного научного студенческого конгресса. 2012: 516-518.

6. Мясоедов А.И., 2018. Инновационные технологии в управлении персоналом / А.И. Мясоедов // В сборнике: Инновационная экономика и менеджмент: Методы и технологии Сборник материалов II Международной научнопрактической конференции. Под ред. О.А. Косорукова, В.В. Печковской, С.А. Красильникова. 2018: 222-224.

7. Мясоедов А.И., 2018. Модели конструктивного управления конфликтами в современных организациях / А.И. Мясоедов // Научный журнал «Дискурс». - 2018. - 2 (16): 96-103.

8. Мясоедов А.И., 2017. Устоявшиеся подходы к организации информационного пространства интернет-СМИ / А.И. Мясоедов // Скиф. Вопросы студенческой науки. 2017. 15 (15): 219-223.

9. Радостева М.В., 2018. К вопросу о про- 
изводительности труда / М.В. Радостева // Научные ведомости Белгородского государственного университета. Серия: Экономика. Информатика. - 2018. - 45 (2): 268-272.

10. Радостева М.В., 2018. Производительность труда: основные тенденции и ключевые факторы развития на современном этапе / М.В. Радостева // Экономика и менеджмент систем управления. - 2018. - 29. - 3-1: 162-172.

11. Романова К.С., 2016. Роль банков на рынке инвестиций/К.С. Романова, А.В. Мирошин//Актуальные проблемы финансового менеджмента. Материалы Международной научно-практической конференции. Институт гуманитарных наук, экономики и информационных технологий; Главный редактор К.В. Ордов; ответственный за выпуск С.В. Шайтура. Бургас, Болгария, 2016. 219-226.

12.Савельева А.В., 2018. Ошибки руководителей в процессе делегирования полномочий в современных организациях / А.В. Савельева, С.П. Иванова // Научный журнал «Дискурс». 2018. - 11 (25): 195-202.

13. Савельева А.В., 2019. Формирование внутреннего кадрового резерва в организации. Этапы и положительные стороны / А.В. Савельева // Бюллетень науки и практики. -2019. - 5. 8: 317-322.

14.Холикова Г.М., 2018. Целевые программы как инструмент государственного и муниципального регулирования / Г.М. Холикова//Бюллетень науки и практики. - 2018. - 4. 12: 404-408.

15. Aikman, D., Haldane, A. and Nelson, B.: 2015, Curbing the credit cycle, The Economic Journal 125, 1072-1109.

16.Behn, M., Detken, C., Peltonen, T. and Schudel, W.: 2013, Setting countercyclical capital buffers based on early warning models: Would it work?, ECB Working Paper Series No. 1604.

17. Bernanke, B., Gertler, M. and Watson, M.: 1997, Systematic monetary policy and the effects of oil price shocks, Brookings Papers on Economic Activity 28, 91-157.

18. Borio, C. (2012). The financial cycle and macroeconomics: What have we learnt? BIS Working Papers No. 395.

19. Borio, C., \& M. Drehmann (2009). Assessing the risk of banking crises - revisited. BIS Quarterly Review, March, pp. 29-46.

20. Breitung, J. and Eickmeier, S.: 2014, Analyzing business and financial cycles using multilevel factor models, Discussion Papers 11/2014, Deutsche Bundesbank, Research Centre.
21.Claessens, S.: 2014, An overview of macroprudential policy tools, IMF Working paper $\mathrm{WP} / 14 / 214$.

22.Claessens, S., Kose, M. and Terrones, M.: 2012, How do business and financial cycles interact?, Journal of International Economics 87, 178190.

23.Comin, D. and Gertler, M.: 2006, Mediumterm business cycles, American Economic Review 96, 523-551.

24.Drehmann, M., Borio, C. and Tsatsaronis, K.: 2012, Characterising the financial cycle: don't lose sight of the medium term!, BIS Working Papers No. 380.

25.Hiebert, P., Klaus, B., Peltonen, T., Schüler, Y. and Welz, P.: 2014, Capturing the financial cycle in the euro area, Financial Stability Review: Special Feature B, 109-117.

26.Kaminsky, G. and Reinhart, C.: 1999, The twin crises: The causes of banking and balance of payments problems, American Economic Review $89,473-500$.

27.Laeven, L. and Valencia, F.: 2012, Systemic banking crises database: An update, IMF Working Paper WP/12/163.

28.Panetta, F. (2013). Macroprudential tools: where do we stand? Remarks during the presentation of the 2013 Financial Stability Review held at the Banque Centrale du Luxembourg.

29.Schularick, M. and Taylor, A.: 2012, Credit booms gone bust: Monetary policy, leverage cycles, and financial crises, 1870-2008, American Economic Review 102, 1029-1061.

\section{References}

1. Ivanova S. P. 2015. Basic mechanisms of anti-crisis management to stabilize the banking system / / Problems of economy, organization and management in Russia and the world. Proceedings of the VIII International scientific and practical conference. 2015. Pp. 75-76. (in Russian)

2. Ivanova S. P., Litvinov A. N. 2016. Performance indicators of real investments: evolution and problems of use / / Bulletin of the Russian economic University.G. V. Plekhanov. 2016. No. 5 (89). Pp. 73-80. (in Russian)

3. Ivanov S. P., Sadykov K. V. 2018. Urgent issues of the activity of the integrated banking structures // Bulletin of the Russian University of Economics named after G. V. Plekhanov. - 2018. No. 1 (97). - Pp. $90-99$ (in Russian)

4. Ivanova S. P., Sadykova K. V. 2017. Features of state corporate and financial control over the activities of integrated banking groups / / Bulle- 
tin of the Plekhanov Russian University of Economics. - 2017. - No. 5 (89). - Pp. 62-71

5. Miroshin A.V. 2012. Factors affecting negative motivation during the crisis / / in the collection: Economic and social problems of the global financial system Collection of articles by participants of the III International scientific student Congress. 2012. Pp. 516-518. (in Russian)

6. Myasoedov A. I. 2018. Innovative technologies in personnel management / A. I. Myasoedov // in the collection: Innovative Economics and management: Methods and technologies Collection of materials of the II International scientific and practical conference. Edited by O. A. Kosorukov, V. V. Pechkovskaya, and S. A. Krasilnikov. 2018. Pp. 222-224. (in Russian)

7. Myasoedov A. I. 2018. Models of constructive conflict management in modern organizations / A. I. Myasoedov / / scientific journal "Discourse". - 2018. - 2 (16). - Pp. 96-103. (in Russian)

8. Myasoedov A. I. 2017. Established approaches to the organization of the information space of Internet media / A. I. Myasoedov / / SKIF. Questions of student science. 2017. No. 15 (15). Pp. 219-223. (in Russian)

9. Radosteva M. V. 2018. On the issue of labor productivity / M. V. Radosteva / / Scientific Bulletin of the Belgorod state University. Series: Economics. Computer science. - 2018. - T. 45. No. 2. - Pp. 268-272. (in Russian)

10. Radosteva M. V. 2018. labor Productivity: main trends and key factors of development at the present stage / M. V. Radosteva / / Economics and management of management systems. - 2018. Vol. 29. - No. 3-1. - Pp. 162-172. (in Russian)

11. Romanova K. S. 2016. the Role of banks in the investment market/K. S. Romanova, A.V. Miroshin / / Actual problems of financial management. Materials of the International scientific and practical conference. Institute of Humanities, Economics and information technology; editor-in-Chief K. V. Ordov; responsible for the issue of S. V. Shaitur. Burgas, Bulgaria, 2016. - Pp. 219-226. (in Russian)

12. Savel'eva A.V. 2018. Mistakes of managers in the process of delegating authority in modern organizations / A.V. Savel'eva, S. p. Ivanova / / scientific journal "Discourse". - 2018. - 11 (25). Pp. 195-202. (in Russian)

13. Saveleva A.V. 2019. Formation of internal personnel reserve in the organization. The stages and the positive side / Savelieva A. // Bulletin of science and practice. -2019. - Vol. 5. - No. 8. Pp. 317-322.
14. Kholikova G. M. 2018. Target programs as a tool of state and municipal regulation/G. M. kholikova / / Bulletin of science and practice. 2018. - Vol. 4. - No. 12. - Pp. 404-408.Aikman, D., Haldane, A. and Nelson, B.: 2015, Curbing the credit cycle, The Economic Journal 125, 10721109. (in Russian)

15.Behn, M., Detken, C., Peltonen, T. and Schudel, W.: 2013, Setting countercyclical capital buffers based on early warning models: Would it work?, ECB Working Paper Series No. 1604.

16.Bernanke, B., Gertler, M. and Watson, M.: 1997, Systematic monetary policy and the effects of oil price shocks, Brookings Papers on Economic Activity 28, 91-157.

17.Borio, C. (2012). The financial cycle and macroeconomics: What have we learnt? BIS Working Papers No. 395.

18.Borio, C., \& M. Drehmann (2009). Assessing the risk of banking crises - revisited. BIS Quarterly Review, March, pp. 29-46.

19.Breitung, J. and Eickmeier, S.: 2014, Analyzing business and financial cycles using multilevel factor models, Discussion Papers 11/2014, Deutsche Bundesbank, Research Centre.

20.Claessens, S.: 2014, An overview of macroprudential policy tools, IMF Working paper $\mathrm{WP} / 14 / 214$.

21.Claessens, S., Kose, M. and Terrones, M.: 2012, How do business and financial cycles interact?, Journal of International Economics 87, 178190.

22.Comin, D. and Gertler, M.: 2006, Mediumterm business cycles, American Economic Review 96, 523-551.

23.Drehmann, M., Borio, C. and Tsatsaronis, K.: 2012, Characterising the financial cycle: don't lose sight of the medium term!, BIS Working Papers No. 380.

24.Hiebert, P., Klaus, B., Peltonen, T., Schüler, Y. and Welz, P.: 2014, Capturing the financial cycle in the euro area, Financial Stability Review: Special Feature B, 109-117.

25.Kaminsky, G. and Reinhart, C.: 1999, The twin crises: The causes of banking and balance of payments problems, American Economic Review 89, 473-500.

26.Laeven, L. and Valencia, F.: 2012, Systemic banking crises database: An update, IMF Working Paper WP/12/163.

27.Panetta, F. (2013). Macroprudential tools: 
where do we stand? Remarks during the presentation of the 2013 Financial Stability Review held at the Banque Centrale du Luxembourg.

28. Schularick, M. and Taylor, A.: 2012, Credit booms gone bust: Monetary policy, leverage cycles, and financial crises, 1870-2008, American Economic Review 102, 1029-1061.
Информация о конфликте интересов: авторы не имеют конфликта интересов для декларации.

Conflicts of Interest: the author has no conflict of interest to declare.

Мясоедов А.И. бакалавр, Московский государственный Психолого-Педагогический Университет (г. Москва, Россия)

Myasoedov A.I. Bachelor, Moscow State University of Psychology and Education (Moscow, Russia) 